\title{
Tangence
}

\section{La lecture et ses troubles : analyse d'un conte fantastique}

\section{Rachel Bouvet}

Numéro 36, mai 1992

La lecture littéraire

URI : https://id.erudit.org/iderudit/025710ar

DOI : https://doi.org/10.7202/025710ar

Aller au sommaire du numéro

Éditeur(s)

Tangence

ISSN

0226-9554 (imprimé)

1710-0305 (numérique)

Découvrir la revue

Citer cet article

Bouvet, R. (1992). La lecture et ses troubles : analyse d'un conte fantastique.

Tangence, (36), 41-51. https://doi.org/10.7202/025710ar d'utilisation que vous pouvez consulter en ligne.

https://apropos.erudit.org/fr/usagers/politique-dutilisation/ 


\section{La lecture et ses troubles: analyse d'un conte fantastique}

\section{Rachel Bouvet}

La lecture occupe une place privilégiée dans les études portant sur les récits fantastiques. Parmi les nombreuses tentatives de définition du genre, celle de Todorov, souvent considérée comme étant l'une des plus importantes, met au premier plan l'hésitation du lecteur entre une explication naturelle et une explication surnaturelle ${ }^{1}$. Pour Lovecraft, c'est le sentiment de peur ressenti par le lecteur qui sert de critère pour affirmer l'appartenance d'un récit au genre fantastique ${ }^{2}$. Caillois, quant à lui, insiste sur l'«impression d'étrangeté irréductible " qui se dégage de ces récits ${ }^{3}$. Quand il ne s'agit pas de définitions à proprement parler, mais de réflexions, d'analyses, les questions relatives à l'attitude du lecteur et à l'effet particulier produit lors de la lecture du fantastique sont fréquemment soulevées. Si les théories du fantastique s'opposent sur plusieurs points, il en est un qui semble faire l'unanimité, même s'il est parfois implicite: c'est le fait que la lecture du récit fantastique possède un caractère singulier, qu'elle diffère des autres lectures. Le trouble ressenti à la lecture de ces textes, les sensations de peur, d'angoisse, ou encore la fascination exercée par le récit, sont en effet des phénomènes importants que l'on peut difficilement contourner. Le problème de la lecture semble donc prédominant et pourtant, quand on regarde de près les différentes théories du fantastique, on ne peut que s'étonner de la disparition de certains de ses aspects essentiels. La question du suspense, par exemple, est très rarement abordée; est-il possible que le récit produise un tel effet sur le lecteur, un effet que l'on pourrait qualifier, lui aussi de fantastique $^{4}$, s'il n'y a pas de suspense? Si le suspense a la faveur des

1 Tzvetan Todorov, Introduction à la littérature fantastique, coll. Points, Paris, Seuil, coll. « Points *, 1970, p. 29.

2 Howard Philip Lovecraft, Épouvante et sumaturel en littérature, Paris, Bourgois, 1969, p. 16.

3 Roger Caillois, Au coeur du fantastique, Paris, Gallimard, 1965, p. 30.

4 L'effet fantastique dont il est question ici est un effet de lecture; il est donc très éloigné des *effets de fantastique - définis par Jean Bellemin- 
lecteurs, il faut admettre qu'il ne suscite pas le même intérêt chez les spécialistes. D'ailleurs, comment cela pourrait-il se faire quand l'objectif privilégié se trouve être la définition du genre ou encore une typologie des récits? En fait, pour pouvoir étudier des phénomènes tels que le suspense, le trouble, l'effet fantastique, il importe avant tout de les replacer dans le cadre où ils se produisent, c'est-à-dire la lecture, qui est par définition un parcours du texte. S'interroger sur la question du suspense, c'est d'abord constater deux choses: d'une part, les essais portant sur cette problématique sont relativement peu nombreux; d'autre part, l'attention y est portée presque exclusivement au cinéma ou au récit policier. On pourrait ajouter qu'un plus grand privilège est accordé au cinéma et il suffit, pour s'en convaincre, de jeter un coup d'œil sur l'Encyclopedia Universalis, qui ne mentionne même pas le récit policier ${ }^{5}$, ou encore sur la définition du Petit Robert: "dans un film, et par extension un spectacle, un récit, moment ou passage de nature à faire naître un sentiment d'attente angoissée".

L'objet de cette étude n'est pas d'étudier le suspense en tant que tel mais de montrer qu'il joue un rôle important dans ce qu'on pourrait appeler l'effet fantastique, qui est un effet de lecture. Quel rapport peut-on établir entre suspense et effet fantastique? Avant de procéder à l'analyse de certains de ces moments de nature à faire naître un sentiment d'attente angoissée, analyse qui permettra d'amorcer une réflexion sur le processus de lecture d'un texte fantastique bien particulier: "Ligeia", d'Edgar Allan $\mathrm{Poe}^{6}$, il est nécessaire de préciser quelques points. On remarque tout d'abord que c'est l'effet produit par le suspense sur le spectateur ou le lecteur qui sert de base à sa définition. Seule la présence d'un "sentiment d'attente angoissée " permet d'affirmer l'existence du suspense. On se trouve donc face à deux types d'effets où se trouve engagée l'affectivité du sujet: le suspense provoque l'angoisse, le fantastique engendre la peur. Même si la

Noël comme étant *un ensemble de procédés, [...] des effets par lesquels le fantastique se rend sensible comme "caractère" (étymologiquement: signe de reconnaissance imprimé au fer rouge sur la peau), comme marque du genre (*Notes sur le fantastique*, Littérature, $\mathrm{n}^{\circ} 8$, décembre 1972, p. 19).

5 L'article consacré au suspense est tiré d'un livre de Jean Douchet sur Hitchcock.

6 Edgar Allan Poe, *Ligeia * [1837], Histoires extraordinaires, traduction de Charles Baudelaire [1856], Paris, Flammation, 1965, p. 279-295. 
définition met sur le même plan le film, le spectacle et le récit, il n'en demeure pas moins que les attitudes en jeu sont fort différentes: le spectateur n'a aucun moyen de contrôler le défilement des images, tandis que le lecteur a tendance à progresser de plus en plus vite dans sa lecture pour se défaire le plus tôt possible de son angoisse. Que le suspense provoque une accélération du rythme de lecture, ce qui est une autre composante de l'effet de suspense, voilà qui est commun à tous les types de récits présentant du suspense. Dans le cas du fantastique, n'est-il pas possible de voir une relation entre cette variation de rythme et l'une des réactions les plus courantes à la peur, c'est-à-dire la fuite? Le récit présente des scènes effrayantes, va-t-on s'attarder avec complaisance sur ces scènes ou bien les fuir au plus vite? Et comment prendre la fuite, quand l'adhésion au discours est tellement forte qu'elle nous empêche de refermer le livre, si ce n'est en se dépêchant de parcourir les lignes ou les pages suivantes? D'un côté l'angoisse, l'accélération du rythme, de l'autre la peur, la fuite en avant. Tous ces éléments sont en étroite relation: l'effet de suspense ne contribuerait-t-il pas à accentuer l'effet fantastique? La tension mise en jeu par le suspense n'est pas uniquement d'ordre affectif: une attente se crée, qui peut tout aussi bien concerner l'apparition d'un événement, le résultat d'une action que la compréhension du récit. Il faut noter à ce sujet que l'on ne se trouve jamais de but en blanc dans un récit fantastique face à un événement étrange: on l'attend avec impatience. Les indices de l'étrange glanés tout au long du récit ne font qu'exacerber l'attente du lecteur. Enfin, le récit fantastique met la compréhension du lecteur à rude épreuve. S'il y a du suspense, c'est que quelque chose nous échappe, que notre désir de savoir nous pousse en avant. Comment ne pas faire un rapprochement avec l'étrange, qui est, par définition, ce qui échappe à la compréhension? C'est sur cet aspect cognitif de la lecture que je me pencherai tout d'abord dans le cadre de cette étude, ce qui m'amènera à remettre en cause la notion d'hésitation développée par Todorov. Le problème de la participation affective du lecteur fera l'objet de la seconde partie, tandis que dans la troisième partie, j'essaierai de montrer que l'on ne peut étudier le trouble lié au suspense sans tenir compte de celui qui se produit à la toute fin du parcours, où l'effet de surprise amène un processus de reconstitution.

Ligeia a des yeux étranges, son érudition est immense, sa passion pour la vie, démesurée. Elle et son mari, qui est le narrateur 
du récit, passent leur temps à lire des ouvrages de métaphysique, qu'il parvient à comprendre grâce aux explications de sa femme. Â la mort de Ligeia, le deuil mélancolique du narrateur cède rapidement la place à un début de folie. Il s'exile en Angleterre, achète une abbaye qu'il décore de façon absolument "fantastique", s'adonne à l'opium et se remarie. Quand Rowena, sa deuxième femme, meurt à son tour, dans des circonstances suspectes, des phénomènes inexplicables se produisent. Occupé à veiller la défunte, et plongé dans les rêveries que lui procure son amour pour Ligeia, le narrateur assiste à un "drame de résurrection ", où un semblant de vie alterne à plusieurs reprises avec la rigidité du cadavre, puis il voit la prétendue morte se lever, ouvrir les yeux: ce sont les yeux de Ligeia.

La mort de Rowena et le drame de résurrection sont deux moments dans le récit où le suspense va grandissant jusqu'à atteindre son paroxysme. Ceci coïncide avec l'apparition de faits insolites. Quand Rowena tombe malade, sa nervosité ne cesse de s'accentuer car elle entend de légers bruits, des soupirs, et remarque des mouvements dans les rideaux. Juste avant qu'elle ne meure, le narrateur a à son tour de curieuses impressions, visuelles et auditives: un frôlement, une ombre, des gouttes rouges qui tombent dans le verre de vin de Rowena, un bruit de pas. Celles-ci sont mises sur le compte de l'hallucination, qui aurait pour cause la maladie en ce qui concerne Rowena, l'opium et un état nerveux ébranlé pour ce qui est de son mari. On nous dit que les deux personnages subissent une déformation de la perception; ceci explique l'étrangeté de leurs sensations. Ce qui est représenté rejoint ce que l'on peut connaître, même sans l'avoir vécu, du délire d'un opiomane ou d'un malade. Comme il ne s'agit pas somme toute d'une situation inexplicable, la présence de ces faits insolites ne devrait pas à première vue poser de problèmes de compréhension au lecteur. Ce sont des faits insolites, soit, mais on les comprend; cela ne veut pas dire pour autant qu'on adhère complètement à cette explication. En effet, il est impossible d'analyser ce passage sans tenir compte de ce qui a précédé dans le parcours du texte. On ne se trouve pas en présence d'un essai sur les délires des malades ou des opiomanes, où un exemple d'hallucination servirait d'illustration aux propos. Au cours de la lecture des pages précédentes, les indices de l'étrange ont été suffisamment nombreux, notamment lors de la description de ligeia, pour que l'on soit à l'affût du moindre phénomène insolite, pour que l'on s'attende à ce que l'étrange 
apparaisse. Dès lors, quand une impression visuelle présente, de façon explicite, les caractères de l'hallucination, cela permet-il d'affirmer que la situation ne pose pas de problèmes de compréhension au lecteur? Celui-ci admet-il d'emblée qu'il ne s'agit que d'une simple hallucination, c'est-à-dire d'une hallucination qui ne masque rien? Malgré les apparences, il y a un véritable problème de compréhension; celle-ci n'est pas parfaite, elle demeure en suspens. D'après Todorov, la réaction du lecteur à ce problème serait l'hésitation. Cette attitude devant le texte est d'ailleurs posée comme étant la première condition du fantastique, qu'il définit ainsi:

[...] il faut que le texte oblige le lecteur à considérer le monde des personnages comme un monde de personnes vivantes et à hésiter entre une explication naturelle et une explication surnaturelle des événements évoqués. ${ }^{7}$

Hésiter entre deux explications ne peut se faire que si on a pris connaissance des deux types d'explication. Or, le conte de Poe n'en présente qu'une seule: l'explication "naturelle", selon laquelle opium, maladie et nervosité excessive provoquent des hallucinations. L'explication surnaturelle ne peut être connue que grâce à une inférence, qui pourrait se résumer ainsi: les soupirs, les ombres, etc., sont les manifestations concrètes de la présence de Ligeia dans la chambre, et celle-ci, sous une forme invisible, se prépare à tuer Rowena avec du poison. Il est bien évident que cette explication ne peut être inférée qu'une fois la lecture du récit achevée. Si le lecteur était à même d'inférer cela à la première lecture, il n'y aurait pas du tout de suspense. Il faut remarquer de plus qu' "hésiter", c'est marquer un temps d'arrêt. Faudrait-il envisager un cas où le suspense, au lieu de favoriser une accélération du rythme de lecture, amènerait au contraire un ralentissement? Il est clair qu'on ne peut hésiter entre deux explications quand on n'en connaît qu'une seule et qu'il ne peut y avoir de suspense quand les deux sont connues. Que le texte impose une attitude particulière au lecteur, cela ne fait pas de doute, mais que cette attitude soit marquée par l'hésitation, voilà qui semble peu fondé. Le jeu entre les deux systèmes d'explication est en fait beaucoup plus complexe que ne l'envisage Todorov; le lecteur s'attend à l'étrange, ce qui l'empêche d'adhérer totalement à l'explication "naturelle " donnée par la narration,

7 Tzvetan Todorov, op. cit., p. 37. 
46

mais peut-on dire qu'il la rejette pour autant? N'est-il pas amené à faire semblant d'y adhérer pour pouvoir continuer sa lecture, quand, dans le fond, il sait très bien qu'il y a autre chose? Le seul moyen de combler cette lacune à la compréhension, c'est de continuer de lire, de se rendre à la fin le plus vite possible pour savoir ce qui se passe. Car ce n'est pas uniquement le besoin de savoir ce qui va se passer qui explique le suspense, c'est également le besoin de savoir ce qui est en train de se passer. On pourrait se demander d'autre part dans quelle mesure l'apparition de faits insolites n'est pas comprise par le lecteur comme étant le début, l'amorce d'un processus ${ }^{8}$. Faire semblant d'adhérer à une explication tout en attendant de connaître la suite, est-ce que cela permettrait d'expliquer en partie le fait qu'un suspense puisse se créer? On a vu que la notion d'hésitation ne semblait pas adéquate pour étudier le processus de lecture. Il faudrait ajouter que le jeu entre les deux types d'explication n'est pas le seul élément sur lequel il faut se pencher lorsqu'on s'interroge sur le déroulement de la lecture.

En regardant de près le récit de la veillée mortuaire, on est à même de constater que certains de ses traits sont problématiques. Le narrateur, seul au chevet de Rowena, voyant le corps changer peu à peu d'aspect, croit tout d'abord que sa femme est tombée dans un sommeil cataleptique. Comme la même scène se répète toute la nuit, on a droit à plusieurs reprises à une description du cadavre, dont voici un exemple:

Le son se fit entendre de nouveau, c'êtait un soupir. Je me précipitais vers le corps, je vis - je vis distinctement un tremblement sur les lèvres. Une minute après, elles se relâchaient, découvrant une ligne brillante de dents de nacre. [...] Il y avait maintenant une carnation imparfaite sur le front, la joue et la gorge; une chaleur sensible pénétrait tout le corps; et même une légère pulsation remuait imperceptiblement la région du cour. (p. 293)

Le passage de la vie à la mort est tout aussi troublant:

8 Dennis Porter affirme dans son article *Backward Construction and the Art of Suspense. (Glen W. Most and William W. Stowe eds, The Poetics of Murder, New York, Harcourt, 1983, p. 328): the *experience of suspension . occurs "whenever a perceived sequence is begun but remains unfinished. (cité par George Dove, Suspense in the Formula Story, Bowling Green, Bowling Green State University Popular Press, 1989 , p. 5). 
Soudainement, la couleur disparut, la pulsation cessa, l'expression de mort revint aux lèvres, et, un instant après, tout le corps reprenait sa froideur de glace, son ton livide, sa rigidité complète, son contour amorti, et toute la hideuse caractéristique de ce qui a habité la tombe pendant plusieurs jours. (p. 293)

Il y a de quoi frissonner à la lecture de ces lignes et il peut être intéressant de chercher à mettre en relation cette réaction provenant d'une émotion avec le problème de compréhension qui se présente au lecteur. Voyons d'abord comment se présente ce problème: si peu d'actions sont représentées dans ce passage, les impressions auditives, visuelles et tactiles sont quant à elles fort nombreuses et vraisemblablement éloignées de ce que le lecteur a pu expérimenter. Le héros entend un soupir? voilà qui semble facile à identifier ${ }^{9}$. Mais, généralement, la perception d'un soupir ne se fait pas dans l'absolu. Elle suppose, entre autres, que quelqu'un a soupiré et que ce soupir a ses raisons d'être: fatigue, tristesse, etc. Ici, la situation est incohérente puisque le narrateur est seul avec un cadavre, qui par définition ne peut pas soupirer. Contrairement à ce qui peut être observé d'habitude, la perception du narrateur ne peut être considérée ici comme étant le point de vue par lequel est décrit l'événement, puisque cette impression auditive constitue un événement à elle seule et se trouve complètement isolée de ce qui pourrait nous permettre de la comprendre. Personne n'a soupiré: le soupir s'est seulement fait entendre... Tout au long de la description du corps, qui est faite de façon fragmentée, on retrouve le même problème de compréhension. Tremblement des lèvres, changement de couleur, chaleur, pulsation du cour: ce sont là des signes de vie, mais il y a toujours une rupture entre ce qui est perçu et ce que devraient présupposer ces perceptions. On n'a guère le choix: la situation est incompréhensible et c'est sur le soupir lui-même, en tant que son, sur le tremblement, bref sur la nature de ce qui est perçu, que se focalise l'attention. Le caractère inexplicable de la situation n'amène pas le lecteur à refermer le livre, ni même à faire une courte pause avant de passer à la suite. Qui, à part Todorov, s'arrêterait sur ces phrases et, impassible, se demanderait: s'agit-il d'une catalepsie ou d'un processus de résurrection surnaturelle? Les questions concernant le pourquoi ou le comment de ces

9 La version originale se présente ainsi: *The sound came again - it was a sigh. Edgar Allan Poe, Poetry and Tales, New York, Literary Classics of the United States, coll. The Library of America, p. 275. 
48

événements se trouvent en fait reléguées au second plan. Passer d'une phrase à une autre, c'est passer d'une impression auditive à une impression visuelle, tactile, et ainsi de suite, impression que l'on ne peut prévoir à l'avance et qui oblige le lecteur à rester à un niveau de compréhension relativement faible. Cette succession de sensations ne peut laisser le lecteur indifférent, étant donné le caractère étrange, voire repoussant, des changements physiques. Que se passe-t-il lorsque l'on est amené à se représenter un cadavre? La représentation du corps demande en effet une attention particulière, c'est ce que souligne Gilles Thérien dans son article "Lire le corps entre les lignes ":

[...] la représentation des corps ne peut se réduire ni à une image précise, ni à une sorte de séquence cinématographique intérieure qui passerait devant les yeux du lecteur. Il s'agit plutôt d'une transformation des données au niveau mental. [...] L'effet de représentation s'appuie sur la perception que le lecteur a de son propre corps, perception interne, et du corps des autres, perception externe médiatisée par son propre corps. ${ }^{10}$

Si la perception interne du lecteur est en jeu, et que les impressions représentées ne se rattachent à rien de connu, on est en droit de se demander si la transformation des données au niveau mental peut réellement se faire sans la participation affective du lecteur. Est-il possible de mettre en relation cette accumulation d'images mentales, qui procède d'une construction inédite, et la réaction émotionnelle qui l'accompagne, qui est de l'ordre de la peur, de l'angoisse? Si ces descriptions du cadavre provoquent une sensation d'incompréhension, elles amènent également à réagir affectivement. Problèmes de compréhension et participation affective sont donc étroitement liés lors de la lecture de ce récit. Ainsi que le fait remarquer Irène Bessière dans son livre $L e$ récit fantastique. La poétique de l'incertain,

[...] les effets émotifs sont d'autant plus marqués que la narration renvoie à une part de silence. Le récit fantastique peut alors se définir comme une technique d'évasion sémantique. ${ }^{11}$

[...] c'est le manque de sens qui effraie, et cette frayeur est tout ce que l'œuvre a à nous proposer. ${ }^{12}$

10 Gilles Thérien, "Lire le corps entre les lignes", Le corps et sa représentation, Montréal, Publications du CIRADE, à paraître.

11 Irène Bessière, Le récit fantastique. La poétique de l'incertain, Paris, Larousse, 1974, p. 184.

12 Ibid., p. 198. 
Cette hypothèse concernant les processus cognitif et affectif de la lecture demanderait à être développée davantage, mais on peut d'ores et déjà se demander quel est son rôle dans la création du suspense. Une tension se met en place à la lecture de "Ligeia" lorsque l'on sent que tout nous échappe. C'est parce qu'on ne sait pas ce qui est train de se passer et que notre réaction aux impressions représentées est avant tout affective qu'on est si pressé de continuer sa lecture. Il est difficile de supporter la description d'un spectacle morbide où un cadavre change de couleur plusieurs fois de suite sans qu'on sache pourquoi; la scène revêt un caractère effrayant. Le seul moyen d'en sortir, c'est de se dépêcher d'arriver aux lignes suivantes, d'accélérer son rythme de lecture. Le jeu qui s'établit entre les processus cognitif et affectif lors de la lecture apparaît donc comme l'un des éléments dont il faut tenir compte lorsqu'on s'interroge sur la création du suspense dans ce récit fantastique.

On ne saurait parler du suspense dans "Ligeia" sans évoquer l'effet de surprise qui attend le lecteur à la toute fin de son parcours. Quand on apprend, dans le dernier paragraphe, qu'il s'agissait bel et bien d'une résurrection et que Ligeia en était l'actrice principale, il n'y a plus de suspense. La lecture a-t-elle fini de nous troubler pour autant? Il est difficile d'imaginer un lecteur qui, rendu au dernier mot, refermerait tranquillement son livre pour s'en aller vaquer à ses occupations. Est-il possible, malgré tout, de considérer la fin du récit comme un moment de détente, en opposition avec la tension qui a précédé? C'est ce que semble vouloir affirmer Jacques Finné, dans son ouvrage $L a$ littérature fantastique. Essai sur l'organisation surnaturelle:

Le récit fantastique est un récit de mystères logiques qui se dissolvent par une explication. [...] Somme toute, le récit se subdivise en deux vecteurs: un vecteur de tension, qui se centre sur les mystères et qui a pour effet de crisper le lecteur; un vecteur de détente, qui annihile la tension. La charnière entre les deux est donnée par l'explication. ${ }^{13}$

Finné ajoute que l'explication peut, selon les récits, être donnée de façon explicite ou présente sous une forme implicite. Dans ce dernier cas, la participation du lecteur est plus intense. C'est ce

13 Jacques Finné, La littérature fantastique. Essai sur l'organisation surnaturelle, Bruxelles, Éditions de l'Université de Bruxelles, 1980, p. 36. 
qui se produit à la lecture de "Ligeia": la clé du mystère n'est pas inscrite noir sur blanc, c'est au lecteur de faire une inférence, qui se traduit inévitablement par un retour en arrière. D'une part, connaître la fin du récit, c'est être à même de dégager certaines données implicites du texte: il n'est jamais clairement dit que Ligeia, au moment de mourir, s'était donné un but, celui de vivre, et qu'elle prendrait les moyens de le réaliser. C'est pourtant ce qu'on est amené à comprendre: il s'agit d'une morte peu ordinaire, capable de tout plutôt que d'accepter une mort définitive. Après avoir tué Rowena, c'est un curieux processus, celui du transfert, de la substitution d'un corps à un autre corps, que Ligeia activera. S'il est possible de retracer l'enchaînement de ces actions lors d'une analyse, ce travail de reconstitution ne se fait pas lors d'une première lecture sans une certaine confusion. Les présupposés qui, dans les passages présentant un suspense, ne pouvaient pas être identifiés, deviennent accessibles, mais sous l'effet de la surprise. Il ne faut pas oublier d'autre part que si ces dernières lignes du texte permettent de comprendre en partie l'enchaînement des événements, elles nous remettent également en présence de l'étrange. Curieusement, les gestes de la personne ressuscitée, qui permettent le dévoilement de son identité, sont les suivants: "se débarrasser du suaire" et "ouvrir les yeux "; deux gestes qui, précisément, se rapportent à un voile. Si l'abandon du suaire permet, par une inférence, d'identifier la chevelure comme étant celle de Ligeia, le mouvement des paupières arrache un cri au narrateur, puisqu'il retrouve les "yeux étranges de son amour perdu " (p. 295). Or, l'élément principal de la description de Ligeia, celui dans lequel toute sa personne semblait se cristalliser, c'était l'étrangeté de ses yeux. La résurrection, manifestation de l'étrange, recoupe donc les indications données précédemment dans le texte. Peut-on dès lors parler de détente, qui viendrait annihiler la tension des pages précédentes? Face à la coïncidence de deux étrangetés, est-il possible de ne pas ressentir un nouveau trouble, puisque là encore quelque chose nous échappe?

Comme on l'a vu au cours de cette analyse, le parcours de "Ligeia" implique une forme de participation particulière, étant donné que, lorsque des situations inexplicables se présentent, il s'agit moins de chercher à comprendre que de ressentir des émotions. La présence d'explications "en trompe-l'œil" dans la narration, l'importance donnée à la représentation d'impressions, le rôle prépondérant de l'effet de surprise, sont quelques-uns des éléments qui permettent de mieux comprendre le déroulement de 
cette lecture. D'autres questions resteraient à poser, telles que le statut particulier de la perception, le rapport entre explicite et implicite, la nature des inférences le long d'un trajet où le suspense implique l'attente, et la fin du récit, le retour en arrière. Enfin, comme la dimension du regard prend d'étranges proportions dans ce conte de Poe, la mise en scène de la lecture a de quoi nous faire réfléchir. À ceux qui ne s'en souviendraient pas, ce récit rappelle que la lecture est avant tout un parcours fait avec les yeux, un parcours qui devient d'autant plus mystérieux quand les yeux en question sont ceux d'une lectrice peu ordinaire. En effet, Ligeia, avant de tomber malade, ne se contentait pas d'expliquer à son mari les livres qu'il ne comprenait pas; le simple fait de poser ses yeux étranges sur les mots qu'il lisait faisait en sorte qu'il comprenne:

Privée du lustre rayonnant de ses yeux, toute cette littérature, ailée et dorée naguère, devenait lourde et saturnienne comme le plomb. Et, maintenant, ces beaux yeux éclairaient de plus en plus rarement les pages que je déchiffrais. (p. 284; je souligne)

Le jeu sur le sens d'“éclairer", qui ici peut vouloir dire à la fois "briller avec éclat" et "rendre intelligible" ne doit pas être pris à la légère ${ }^{14}$, puisqu'à vrai dire le narrateur n'est pas le seul à avoir des problèmes de compréhension. Comment oublier ceux qui se présentent au lecteur lors de son parcours du texte? De même qu'il est apparu impossible d'étudier "Ligeia " sans tenir compte du déroulement de la lecture, marquée par le suspense, il semble maintenant difficile de se contenter d'hésiter sur le sens de ce récit. Plutôt que de vouloir à tout prix lui donner un sens définitif - puisque c'est là finalement ce vers quoi tend l'hésitation -, il serait peut-être plus intéressant de chercher à montrer que, pour un lecteur, c'est en l'espace d'un clin d'œil que le sens peut devenir obscur...

14 Cette ambiguité est propre à la traduction de Baudelaire. En effet, dans la version anglaise, où le verbe "to shine* est employé, on ne retrouve pas ce jeu sur les deux façons possibles d'*éclairer * un texte: *Wanting the radiant lustre of her eyes, letters, lambent and golden, grew duller than Saturnian lead. And now those eyes shone less and less frequently upon the pages over which I pored." (Edgar Allan Poe, Poetry and Tales, op. cit., p. 266-267, je souligne). Cet effet de traduction contribue à renforcer l'aspect ambigu du conte de Poe. 